\title{
ENSAIO DE OPERACIONALIZAÇÃO DE INDICADORES DE DESENVOLVIMENTO SUSTENTÁVEL POR MODELAÇÃO GEOGRÁFICA
}

Paulo Morgado ${ }^{1}$

\begin{abstract}
Resumo - Este artigo apresenta uma proposta de operacionalização do conceito de desenvolvimento sustentável, abordado frequentemente numa perspectiva teórica, por recurso aos Sistemas de Informação Geográfica (SIG). O exercício a que aqui se alude é apenas a parte operativa de um projecto mais vasto que pode resumir-se a três fases. 1) Pesquisa - definição e esclarecimento do conceito de desenvolvimento sustentável, definição dos indicadores, determinação do enquadramento teórico e estrutura metodológica. 2) Operacionalização - identificação e aquisição de informação pertinente, edição e validação da informação, criação de um projecto SIG, definição e construção de indicadores de desenvolvimento sustentável, adaptação e concepção de um modelo geográfico. 3) Análise - recolha e interpretação dos resultados à luz das teorias defendidas por alguns autores de referência. Para a realização do exercício, assim como para o desenvolvimento dum modelo causal, o uso dos SIG foi imprescindível, enquanto instrumento de excelência para aquisição, armazenamento, manipulação, gestão e visualização de significativos volumes de informação de natureza geográfica. A área metropolitana de Lisboa foi o território escolhido para aplicação do modelo.
\end{abstract}

Palavras-chave : Sistemas de Informação Geográfica, modelação geográfica, desenvolvimento sustentável, indicadores, ordenamento e planeamento do território.

\begin{abstract}
Practice of Sustainable development indicators by Geographic Modelling. This article presents a proposal of how to put into practice the sustainable development concept, usually strictly theoretical, by using the Geographic Information System (GIS). This exercise is only the technical side of a more extensive project that can be summarized into the following three stages. 1) Research - definition and clarification of sustainable development concept, definition of indicators, determination of a theoretical and methodological framework. 2) Operational methods - identification and acquisition, editing and validating information, creating a GIS project, definition and creation of sustainable development indica-
\end{abstract}

1 Investigador do Centro de Estudos Geográficos e Assistente no Departamento de Geografia da FL/UL. E-mail: pms@fl.ul.pt 
tors, adaptation and construction of a geographic model. 3) Analysis - collection and interpretation of the geographic model outcomes by comparing them with theories forwarded by leading researchers. In order to carry out the exercise and to develop a conceptual methodology of a casual model, GIS has proved to be a fundamental instrument in order to acquire, integrate, manipulate, manage and visualize geographic information. The model was applied to the Lisbon metropolitan area.

Key words: Geographic Information System, geographic modelling, sustainable development, sustainable development indicators, land use planning and management.

Résumé - EsSAI D'opéraTiONNALISATION D'INDICATEURS DE DÉVELOPPEMENT DURABLE PAR MODÉLISATION GÉOGRAPHIQUE. La notion de développement durable est fréquemment abordée dans une perspective uniquement théorique. Nous proposons de l'envisager sous un angle opérationnel, en ayant recours aux SIG. Le présent exercice est une composante d'un projet plus vaste, divisé en trois phases: 1) Recherche - définition du concept de développement durable et de ses indicateurs, délimitation du cadre théorique et de la démarche méthodologique. 2) Opérationnalisation - identification et acquisition d'information pertinente, édition et validation de l'information, création d'un projet SIG, définition et création d'indicateurs de développement durable, adaptation et conception d'un modèle géographique. 3) Analyse - interprétation des résultats obtenus en fonction des théories défendues par divers auteurs. Le recours aux SIG, en tant qu'excellents instruments d'acquisition, de stockage, de manipulation, de gestion et de visualisation d'une information géographique volumineuse, s'est révélé essentiel pour la réalisation de cet exercice et le développement d'un modèle causal, appliqué à l'aire métropolitaine de Lisbonne.

Mots-clés - Systèmes d'Information Géographique, modélisation géographique, développement durable, indicateurs, aménagement et développement du territoire.

\section{INTRODUÇÃO}

Iniciada no decurso da II Guerra Mundial, a utilização da informática permitiu um forte avanço nas ciências sociais, humanas e exactas. Desde então, métodos, técnicas e tecnologias foram reconsiderados, revistos e reformulados, para a optimização dos processos de manipulação e tratamento de informação geográfica, e para a produção de mais e melhor conhecimento da realidade. Foi desse período que emergiu uma confluência de conhecimentos geográficos teóricos e de renovadas técnicas para análise espacial, como sejam os Sistemas de Informação Geográfica (SIG).

Os órgãos de soberania, que têm a responsabilidade de ordenar e gerir o território, reconhecem a dificuldade em aferir e conjugar a quantidade enorme 
de dados com que são confrontados e a multiplicidade de perspectivas pelas quais se pode abordar o território. Neste contexto, é exigida aos técnicos capacidade de diagnóstico, análise, propostas e soluções, que permitam melhores formas de sistematizar a informação. Os SIG possuem a capacidade de estabelecer conexões entre volumosas bases de dados e determinar, com exactidão, localizações mediante a atribuição de coordenadas terrestres. Este simples facto permite visualizar de forma dinâmica o posicionamento geográfico, relativo e absoluto, de temas que têm sido discutidos de forma pouco integrada, e consequentemente não-operacionalizados. Com recurso aos SIG, é possível a modelação do conceito de desenvolvimento sustentável, a sua medição numa escala de valores, e a sua visualização por invocação de cenários.

\section{INDICADORES DE DESENVOLVIMENTO SUSTENTÁVEL}

A etimologia da palavra «indicador» é originária do latim indicatore, que significa algo a salientar ou a revelar. Os Indicadores de Desenvolvimento Sustentável (IDS) formam um conjunto estatístico, simples ou em conjugação, que, quando avaliados e devidamente analisados, possibilitam a visualização fiável dos sistemas sobre os quais tomamos decisões. Nesta medida, os indicadores são sempre um reflexo de valores, objectivos ou subjectivos, de acordo com o indivíduo, grupo, ou sociedade, que os elegeu.

We live by indicators. A smile signals friendliness, a gray sky - possible rain, a red traffic light-danger of collision, the hands of a watch - the time of a day, a high body temperature - illness, rising unemployment - social trouble. (...) If we want to compare future paths and their impacts, we have to look at indicators that provide relevant information about future developments (Bossel, 1998:70). De uma forma intuitiva, todos usamos indicadores para modelar ou sustentar conceitos e/ou ideias percepcionadas de sistemas complexos.

Pode dizer-se que a inclusão dos indicadores nas principais figuras de planeamento representa a atribuição de uma componente prática e quantificada ao conceito de desenvolvimento sustentável. Significa isto que os IDS permitem, quando devidamente instrumentalizados pelas Tecnologias de Informação Geográfica (TIG), e em particular pelos SIG, operacionalizar o conceito de desenvolvimento sustentável, contribuindo para a implementação dos princípios na base do desenvolvimento sustentável e promovendo o compromisso entre os sistemas sociais, económicos, e ambientais, em prol da qualidade de vida.

\section{A MODELAÇÃO COMO FORMA DE OPERACIONALIZAÇÃO DO CONCEITO DE DESENVOLVIMENTO SUSTENTÁVEL}

A modelação tem sido um caminho útil para testar hipóteses, teorias e princípios. Os modelos consistem em simplificações e generalizações da realidade, a partir das quais se podem fazer formulações mediante recurso a formalismo matemático. Segundo Odum, as operações dos modelos por computador permitem 
prever resultados prováveis, à medida que no modelo se vão alterando parâmetros, se juntam outros ou se retiram os anteriores... (1971: 9). É neste quadro que se assume a modelação dos IDS, como a forma mais expedita e sólida, para operacionalizar o conceito de desenvolvimento sustentável.

A criação e desenvolvimento de um processo de modelação deve iniciar-se através de um conjunto de observações, que são posteriormente transcritas para uma estrutura de entidades e relações. Procedendo-se depois à sua conversão para uma linguagem matemática, que torne passível a sua computação, isto é, a criação de um programa que pode ser compilado e 'corrido' como uma simulação (SILVA, 2002).

A realidade pode traduzir-se em informação geográfica, a partir da qual se formulam interrogações e hipóteses. É neste âmbito que a modelação geográfica surge como um vértice das recentemente designadas Ciências de Informação Geográfica $^{2}$ (CIG) e da produção de conhecimento sobre fenómenos espaciais (fig. 1), em que o recurso a métodos e técnicas avançadas permite fazer simula-

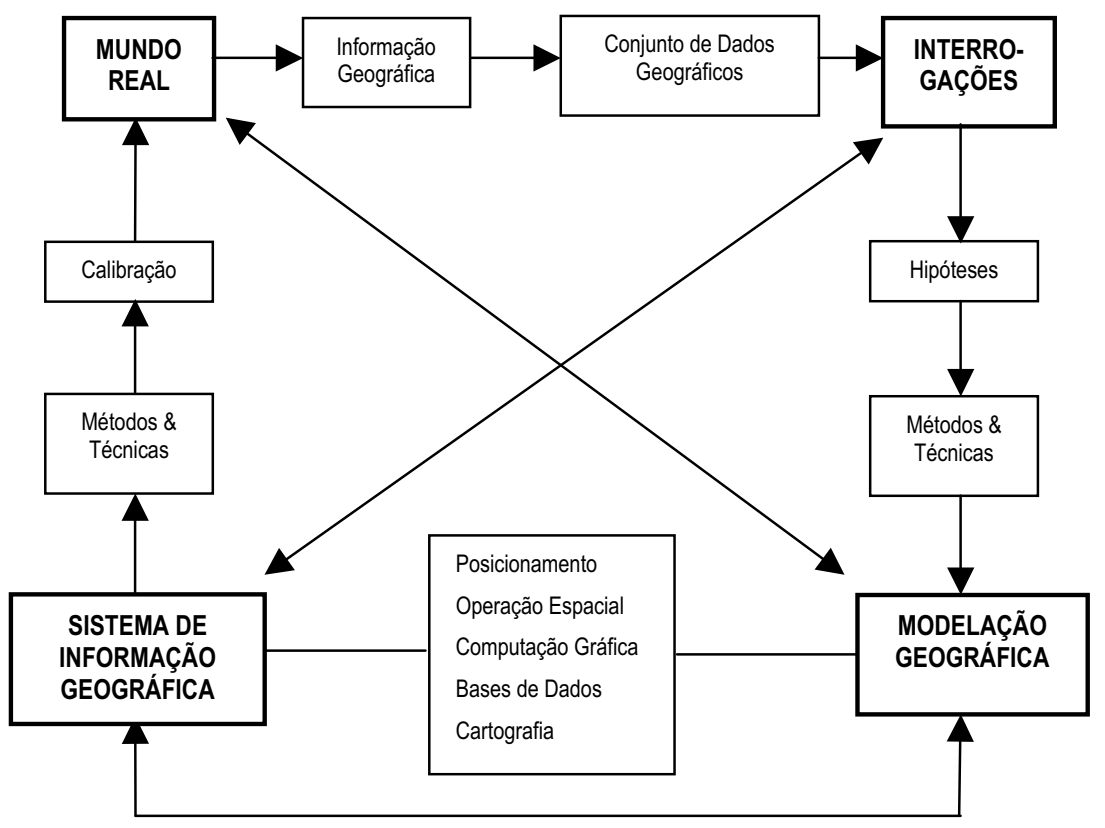

Fig. 1 - Estruturação das componentes das CIG.

Fig. 1 - Relationships among the components of the Geographic Information Sciences.

2 O termo Ciências de Informação Geográfica foi pela primeira vez publicado num artigo, em 1992, por Michael Goodchild, onde este afirma que as CIG estudam as questões fundamentais àcerca da informação geográfica (geodesia, as ciências cognitivas, estatística e a geoestatística, entre outras) e investigam a forma de resolver os problemas complexos da Humanidade, por meio de uma ferramenta priveligiada: os SIG. 
ções da realidade e confrontar o resultado com o mundo observado para verificar se as hipóteses inicialmente estabelecidas se comprovam. Em caso afirmativo, o modelo conseguido é aceite, uma vez que permitiu a resolução de um problema; em caso negativo, o modelo terá de ser calibrado, revisto e alterado, em função de novas condições iniciais.

O tempo (data) e o espaço (coordenadas) são atributos comuns a todos os IDS; característica vulgar que os torna georreferenciáveis e, por conseguinte, passíveis de modelação. Todavia, não basta criar um modelo. É preciso que aquele seja capaz de integrar, manipular, cruzar, classificar, medir e disponibilizar extensos volumes de informação geográfica. Em suma, modelos dinâmicos, fiáveis e 'democráticos' orientados para o utilizador comum.

\section{ENSAIO DE MODELAÇÃO GEOGRÁFICA DE IDS}

A escolha do modelo de dados para representar e operacionalizar os IDS é crucial, quer para a viabilidade, quer para fidelidade do modelo. No âmbito dos SIG, consideram-se dois tipos fundamentais de modelos conceptuais ${ }^{3}$ : o SIG Vectorial e o SIG Raster.

De acordo com o objectivo do presente ensaio, o tipo de informação a manusear, o equipamento e programas disponíveis e o recurso à análise espacial em SIG Raster imperou, pelo facto das estruturas de representação deste modelo apresentarem vantagens para operar fenómenos de natureza espacial contínua. Numa primeira fase, na modelação geográfica dos IDS fez-se a preparação e a estruturação dos temas recorrendo-se ao modelo relacional; e, numa segunda fase, evoluiu-se para um modelo raster orientado a objectos, assim como para a definição de algumas etapas processuais, nomeadamente na criação de um interface de utilização 'amigável'. Em suma, desenvolveu-se um sistema modelar híbrido com o intuito de adaptar o paradigma aos dados e não o inverso, tal como é característico dos SIG ditos convencionais que utilizam Sistemas de Gestão de Bases de Dados (SGBD), geralmente do tipo relacional.

\section{Modelo Causal}

O modelo concebido é flexível, pois permite a manipulação da informação por substituição, alteração e introdução de novos dados, sempre sem condicionalismos de escala. A abordagem ao conceito de desenvolvimento sustentável passa pela computação de um conjunto de dados geográficos, que traduzem os efeitos das pressões sobre o território dos quais nos ocuparemos, com a finalidade de estabelecer medidas avaliativas. Neste sentido, assumiu-se a fase com-

3 Dos SIG conceptuais diz-se serem aqueles que derivam, ou se classificam com base num determinado conceito. Sobre isto é referido que «A concretização de um modelo geográfico conceptual pode designar-se por Sistema de Informação Geográfica» (MATos, 2001: 55). 


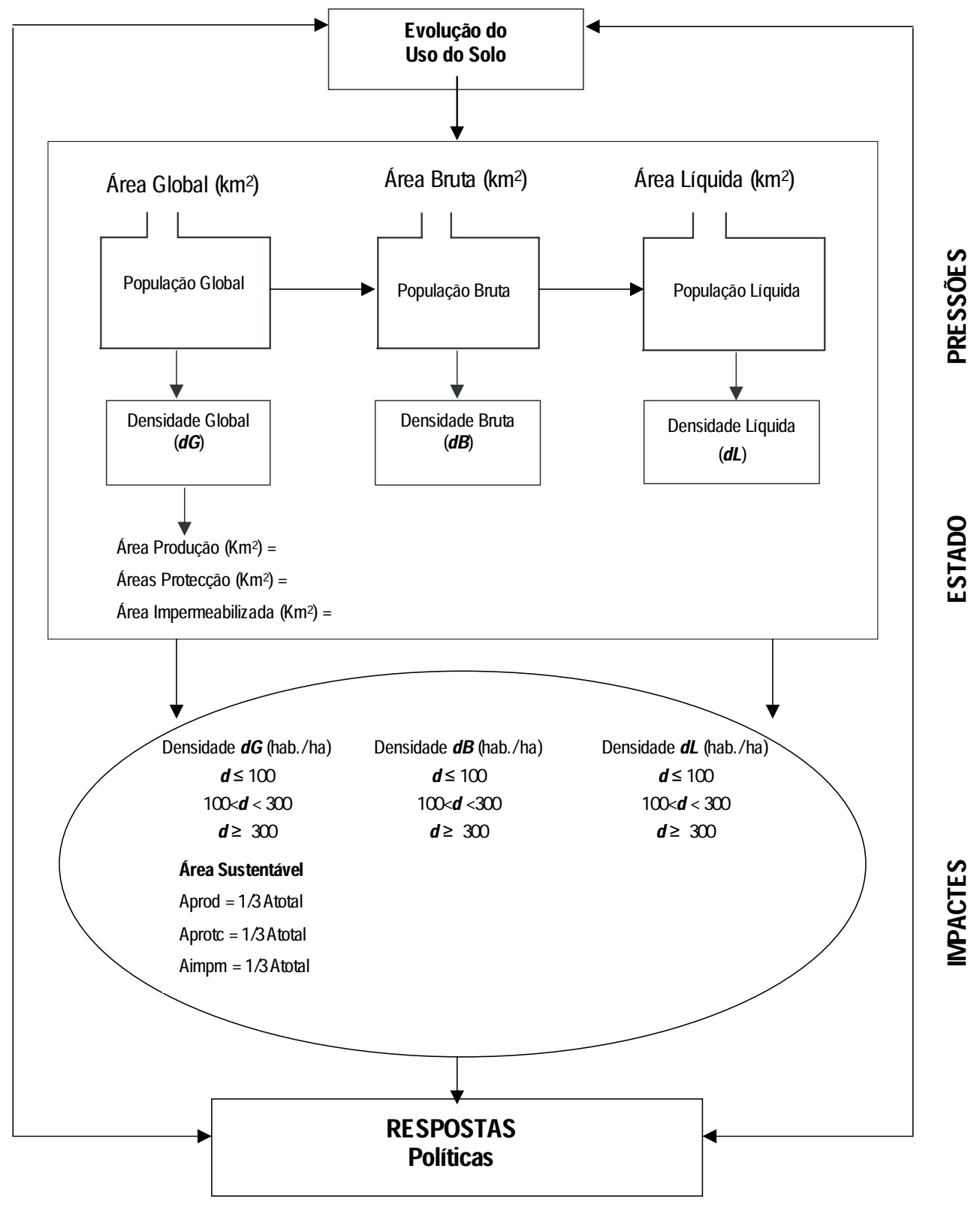

Fig. 2 - Estrutura do modelo causal para a AML.

Fig. 2 -Structure of the cause-effect model for the Lisbon Metropolitan Area. 
putacional como um suporte de um sistema de Base de Dados orientado por objecto, para realização de uma interface gráfica que se designou de Medidor de Impactes.

Aplicado ao caso de estudo, desenvolveu-se um modelo causal com incorporação do conceito de desenvolvimento sustentável, com base em trabalhos desenvolvidos por autores consagrados, em que se exprimem os princípios do equilíbrio de usos do solo e da pressão populacional sobre determinados espaços geográficos.

A descrição da estrutura conceptual do modelo causal (fig. 2) é a seguinte:

Área Global: corresponde à área do território em estudo, calculada com base na junção das áreas impermeabilizadas, com as áreas de produção, e as áreas de protecção (cf. definições à frente). De notar que o cálculo para determinação desta área é resultado de uma operação lógica de união (Quadros I e II).

Área Bruta: corresponde à subtracção das áreas de protecção à área global.

Área Líquida: subtrai, à área bruta, as áreas de produção. Como resultado tem-se uma coincidência espacial e conceptual entre o que se entende por área líquida e áreas de impermeabilização, ou seja a porção de território sujeita às maiores acções antrópicas.

Impactes: resultado do confronto entre o estado e os quadros de referência, onde são definidos os valores indicadores de factores primários de qualidade de vida, mormente os valores da densidade populacional em espaço urbano, e os valores que contribuíram para o que se designou por área sustentável. Dos resultados apurados pelo cálculo das áreas fica-nos a medida exacta do (des)equilíbrio relativamente ao que é aceitável para uma área sustentável, ou por um espaço urbano bem, razoavelmente, ou mal ocupado, de acordo com a densidade que lhe está adstrita.

Densidades: medida que reflecte uma perspectiva da qualidade de vida em meio urbano. Segundo as medidas definidas por SERRATOSa (2000), tem-se bom nível de vida urbano uma densidade até 100hab./ha, razoável entre $100 \mathrm{e}$ 200 hab./ha e mau mais de 300hab./ha (fig. 3).

Área Sustentável: corresponde, segundo as normas definidas por $\mathrm{F}_{\mathrm{ABOS}}{ }^{4}$ (1985: 20-43), a uma repartição equilibrada ${ }^{5}$ da área global pelas três categorias de uso do solo, cada uma com $1 / 3$ da área global.

4 Trata-se de uma tese que foi recuperada das ciências biológicas e ecológicas para o domínio do ordenamento e do planeamento do uso do solo. Baseava-se no modelo de classificação do solo em quatro componentes dependendo da sua função no ecossistema regional: Espaço de Protecção; Espaço de Produção; Espaço Urbano-Industrial e o Espaço Multi-Uso ou de Compromisso.

5 Uma clara analogia com a Segunda Lei da Termodinâmica, relacionada com o princípio da estabilidade. De acordo com este conceito, qualquer sistema natural isolado, com energia a atravessá-lo, trate-se da própria Terra ou de uma entidade menor, como por exemplo um lago, tende a alterar-se até que se desenvolva um ajustamento estável, com mecanismos de auto-regulação (Odum, 1971: 57). 
Respostas: uma vez ponderados os outros estádios do modelo, cabe ao decisor a tomada de responsabilidade pelas medidas a implantar por forma a se resolverem problemas e/ou a minorarem situações deficientes, com o objectivo de proporcionar um bom nível de qualidade de vida às populações.

As respostas podem ser apresentadas sob a forma de orientações para o ordenamento do território, num determinado horizonte temporal (entre 5 a 10 anos), onde os vários sistemas sociais, económicos e ambientais são integrados. Caso o modelo indique uma situação sustentável, o ciclo permanecerá em estado de monitorização; caso o modelo declare uma situação insustentável, medidas políticas deverão ser accionadas para que o equilíbrio seja alcançado, ou seja, reinicia-se o ciclo do modelo causal. Em qualquer das situações, as respostas nunca deverão ser entendidas como fase terminal; antes pelo contrário, deverão ser sempre uma fase processual do ciclo, isto é, do modelo.

\section{Metodologia}

Para a concretização deste modelo, e antes de se iniciar a sua computação, efectuam-se alguns procedimentos, nomeadamente a esquematização das análises espaciais e respectiva modelação. Sintetizando, têm-se dois estádios distintos, quer do ponto de vista computacional, quer do ponto de vista temporal; o que resta em comum é o território de experimentação do modelo: a área metropolitana de Lisboa.

No primeiro estádio, o processamento do conjunto de dados geográficos referentes à situação avaliadora das densidades populacionais, em que a estrutura SIG apenas considera os temas: Área (Global, Bruta e Líquida) e População (Global, Bruta e Líquida). No segundo estádio, o factor tempo assume destaque e conduz a duas novas situações:

- Situação de Referência: inventário do uso do solo. Diz respeito à informação geográfica obtida no projecto CarTus/AML90 ${ }^{6}$.

- Situação Oficial: PROTAML92 - Plano Regional de Ordenamento do Território da área metropolitana de Lisboa. As operações de análise espacial baseiam-se em informação geográfica de uso do solo decorrente da proposta da carta de ordenamento do plano em causa.

Acresce ainda a necessidade de se efectuarem operações de análise espacial para cada um das seguintes situações:

- Áreas de Produção - as operações desenvolvidas servem para determinar, segundo os usos do solo que lhe estão adstritos, espaços que suportam recursos primários essenciais à vida, por exemplo, espaços agrícolas.

6 Projecto resultante de um protocolo entre o Instituto de Dinâmica do Espaço (Dep. GPR da FCSH/UNL) e a Área Metropolitana de Lisboa, cuja metodologia envolveu, no seu arranque, a elaboração da matriz classes/critérios e trabalhos de foto-interpretação, de campo, e de digitalização; posteriormente os trabalhos consistiram na criação de projecto MGE GIS Environment, para cálculo de áreas e cruzamentos com os dados dos Censos de 1991. 
- Áreas de Protecção - as operações desenvolvidas servem para determinar, segundo os usos do solo que lhe estão adstritos, espaços que devem ser preservados e perpetuados em prol da qualidade de vida das populações.

- Áreas de Impermeabilização - as operações desenvolvidas servem para determinar, segundo os usos do solo que lhe estão adstritos, espaços resultantes maioritariamente de acções antrópicas.

O cálculo das áreas decorrentes dos processos de cruzamento de informação geográfica permite não só a verificação da repartição de usos do solo na AML, como também o confronto entre uma situação de referência - o inventário de uso do solo - e uma situação oficial, baseada nas propostas do PROTAML92 - prospectiva.

Ainda relativamente ao exercício para modelação espacial do conceito teórico de desenvolvimento sustentável com base nos indicadores (população residente e solo urbano), convém clarificar a posição aqui defendida da escolha dos indicadores, face a teorias que alguns autores vem defendendo e discutindo, nomeadamente a questão da qualidade de vida das populações em meio urbano, em particular, nas áreas metropolitanas. Efectivamente vem sendo apontada a necessidade de se estabelecerem limites à expansão do fenómeno urbano (FABOs, 1985; Serratosa, 2000; Telles, 2000; Мachado, 2000), assim como se tem alertado para a importância da criação de espaços verdes como factor de equilíbrio no contínuo urbano.

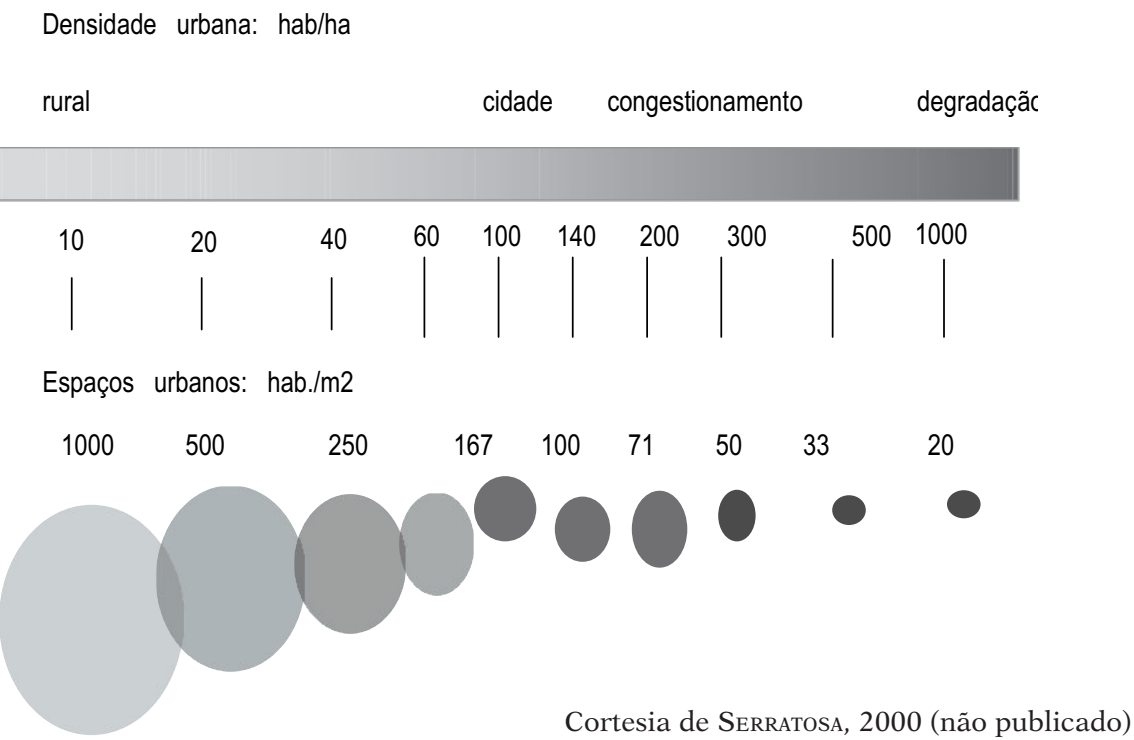

Fig. 3 - Espectro dos factores primários de qualidade de vida urbana.

Fig. 3 - Primary factors affecting the urban quality of life. 
Sobre esta questão dos espaços verdes urbanos, sublinhe-se a distinção entre parques urbanos e áreas protegidas, normalmente localizadas nas imediações da urbe, aspecto apontado à metrópole de Barcelona, mas que pode ser facilmente adaptado a outras áreas metropolitanas da Europa, incluindo Lisboa: According to the global diagnosis of the metropolitan area, there are too many protected natural areas (nature parks and reserves) forest parks and agricultural zones on the boundaries of the urban centres; what is lacking is an intermediate level of protection (protected agricultural areas, metropolitan corridors, etc.) which might confer continuity and consistency on a integrated system of open spaces, as opposed to a disconnected string of isolated areas with more or less legal protection (SERRatosa, 2000). Estas palavras colocam ainda um outro ponto de discussão, que é o carácter legal e proteccionista que deve ser instituído para as áreas verdes intersticiais, fundamentais para a qualidade de vida em meio urbano.

Para a concretização e obtenção dos valores foi necessária a estruturação de um esquema processual de fluxos e prioridades com base no cruzamento da informação geográfica seleccionada. Estes processos designam-se por Sistema de Informação Geográfica e têm como normas de esquematização as apresentadas na figura 4:

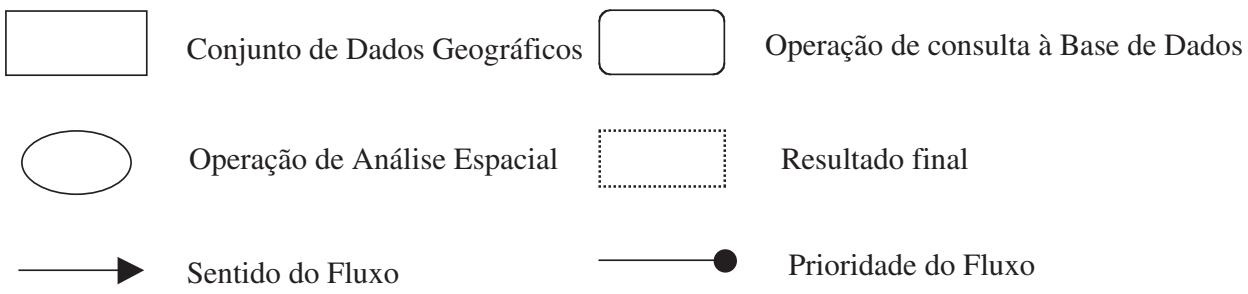

Fig. 4 - Normas de representação de esquemas em SIG.

Fig. 4 - Standard rules for presenting GIS schema.

Essas normas traduzem-se nos esquemas processuais do SIG aplicado ao desenvolvimento sustentável para a AML, da forma que a seguir se apresenta nas figuras 5 a 9 :

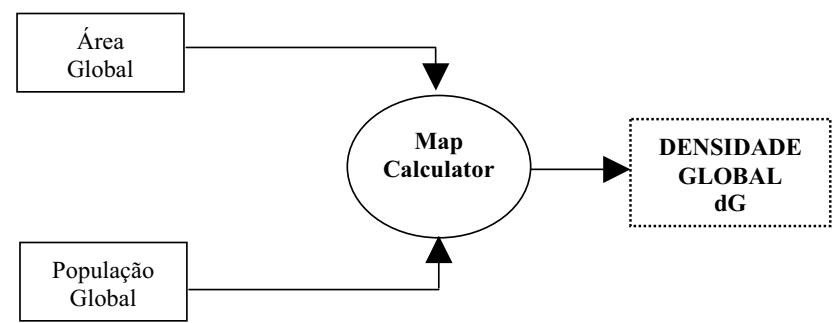

Fig. 5 - Densidade populacional global.

Fig. 5 - Global population density. 


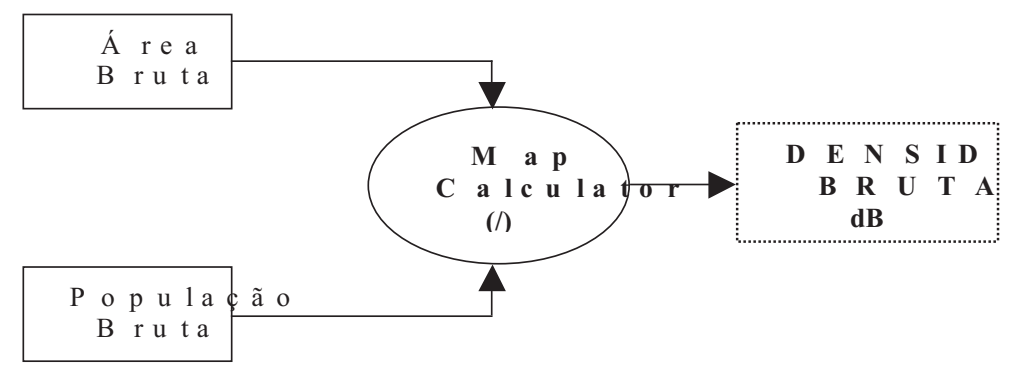

Fig. 6 - Densidade populacional bruta.

Fig. 6 - Gross population density.

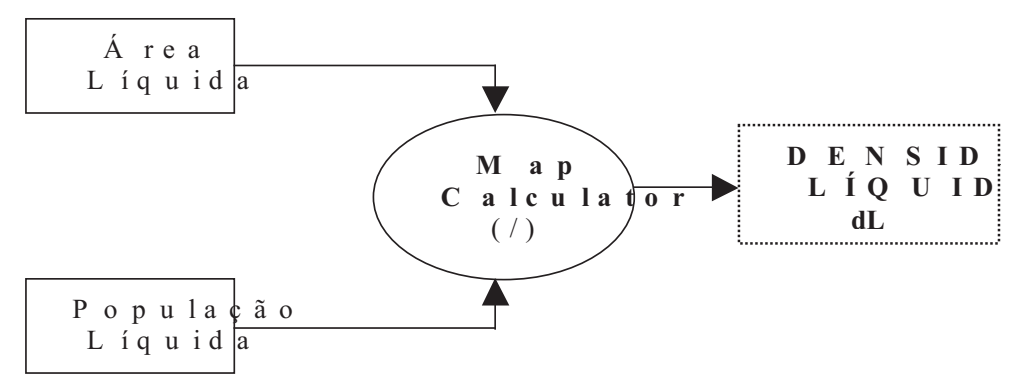

Fig. 7 - Densidade populacional líquida.

Fig. 7 - Net population density.

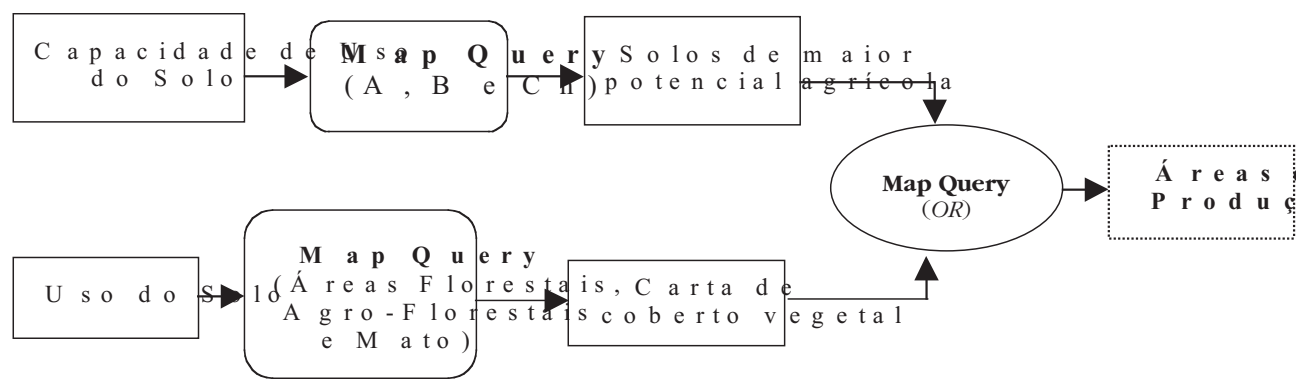

Fig. 8 - Área Global - Processo para determinação das áreas de produção.

Fig. 8-Global Area - Process to obtain landscape areas of productivity. 


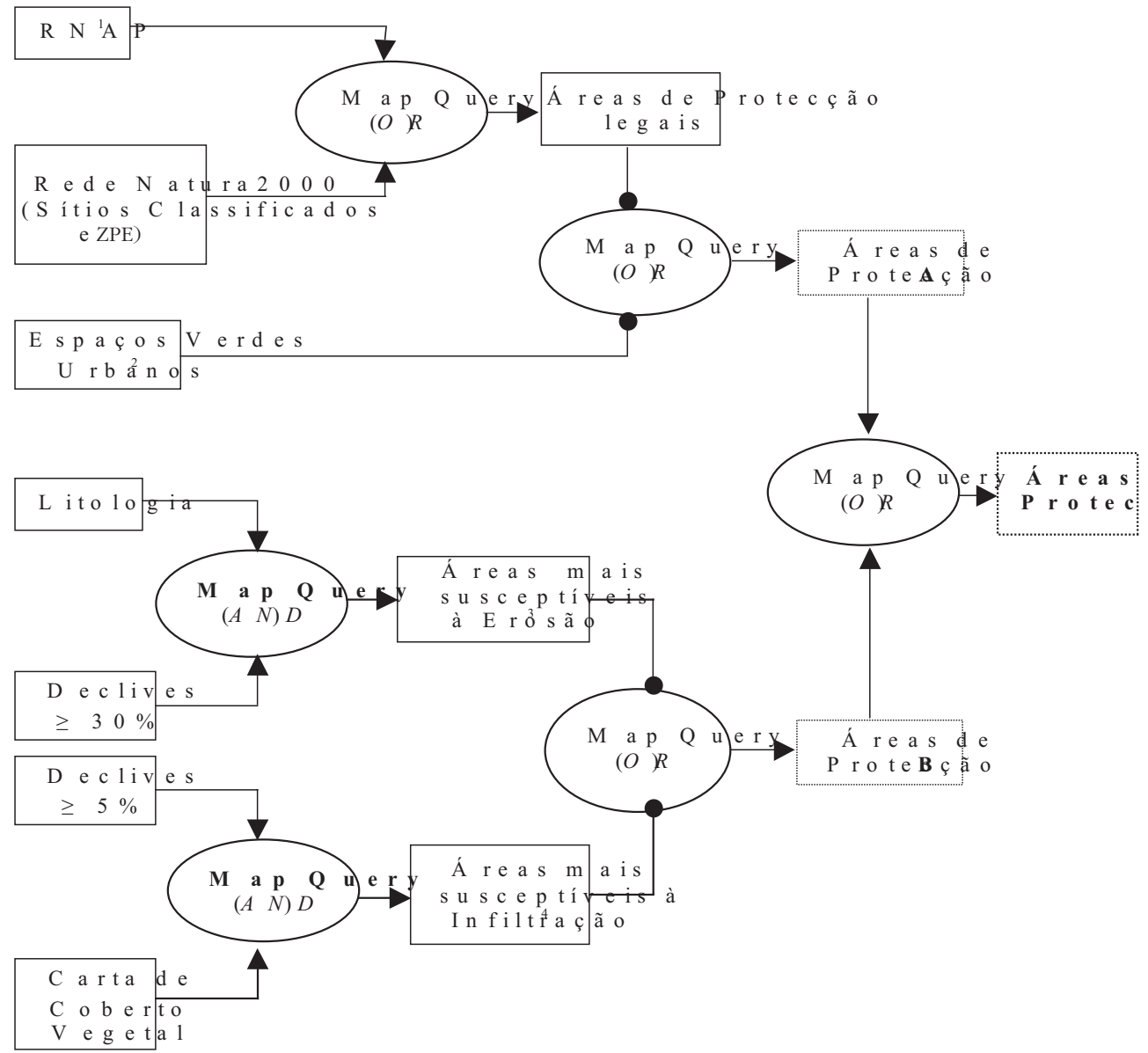

Fig. 9 - Área Global - Processo para determinação das áreas de protecção.

Fig. 9 - Global Area - Process to obtain areas of protection.

\section{Legenda da figura 9:}

1 Rede Nacional de Áreas Protegidas;

2 Obtidos por inquirição ao tema de Uso do Solo de 1990.

3 Adaptado de Mopta, 1995: 573 (matriz definidora do mapa geomorfológico). Reflecte o grau de erodibilidade do solo em função dos declives do terreno.

4 Adaptado de Морта, 1995: 573 (matriz definidora do mapa de protecção do solo pela vegetação). Reflecte o grau de infiltração do solo em função dos declives.

A - Inclui as áreas legalmente protegidas e aquelas que deveriam ser.

B - Inclui as áreas cujas geomorfologia e uso do solo, tornam mais sensíveis. 


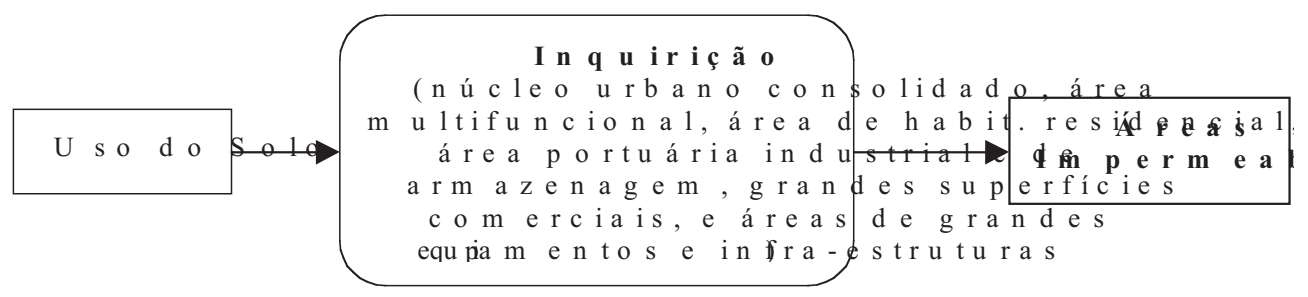

Fig. 10 - Área Global - Processo para determinação das áreas impermeabilizadas. Fig. 10 - Sub-area of the Lisbon Metropolitan Area corresponding to the parishes with a population density greater than 1000inb. $/ \mathrm{km}^{2}$ (2001).

Os seguintes passos processuais (área produção, área protecção e área de impermeabilização) serão corridos conforme estrutura acima explanada, apenas com a diferença da classificação de uso do solo segundo o PROTAML92.

\section{Resultados: análise crítica}

Após a conclusão dos processos de cálculo das áreas, o passo seguinte consiste no cruzamento do valor das áreas obtidas com o contigente de população residente. Este cruzamento repete-se para as duas margens da AML, por forma a obterem-se valores indicadores de pressão. Recorrendo a operações de álgebra, extraíram-se os seguintes resultados (Quadro I):

Quadro I - Valores das áreas e respectivas densidades populacionais, calculadas segundo o Uso do Solo de 1990.

Table I - Area dimensions and population densities, by Land Use, 1990.

\begin{tabular}{|l|c|c|c|c|c|c|}
\cline { 2 - 8 } \multicolumn{1}{c|}{} & \multicolumn{9}{c|}{$\left(\mathbf{k m}^{\mathbf{2}}\right)$} \\
\cline { 2 - 8 } \multicolumn{1}{c|}{} & AML Norte & $\mathbf{\%}$ & AML Sul & $\mathbf{\%}$ & $\mathbf{A M L}$ & $\boldsymbol{\%}$ \\
\hline Área de Produção & 526,6 & 60,8 & 795,3 & 75,8 & 1321,9 & 69,0 \\
\hline Área de Protecção & 278,8 & 32,2 & 682 & 65,0 & 960,8 & 50,2 \\
\hline Área Impermeabilizada & 241,1 & 27,8 & 129,3 & 12,3 & 370,44 & 19,3 \\
\hline Área Global (valor de referência) & $\mathbf{8 6 6 , 1}$ & $\mathbf{1 0 0}$ & $\mathbf{1 0 4 9 , 1}$ & $\mathbf{1 0 0}$ & $\mathbf{1 9 1 5 , 2}$ & $\mathbf{1 0 0}$ \\
\hline
\end{tabular}

\begin{tabular}{|c|c|c|c|c|c|}
\hline & \multicolumn{5}{|c|}{ Área Global } \\
\hline & Habitantes & ha & Densidade Global $\left(\mathrm{hab} / \mathrm{km}^{2}\right)$ & Densidade Global (hab/ha) & Total AML \\
\hline AML Norte & 1874497 & 86607,32 & 2164,36 & 21,64 & \multirow{2}{*}{27,77} \\
\hline \multirow[t]{3}{*}{ AML Sul } & 643106 & 104918,24 & 612,95 & 6,12 & \\
\hline & \multicolumn{5}{|c|}{ Área Bruta } \\
\hline & Habitantes & ha & Densidade Bruta $\left(\mathrm{hab} / \mathrm{km}^{2}\right)$ & Densidade Bruta (hab/ha) & Total AML \\
\hline AML Norte & 1874497 & 77706,88 & 2412,26 & 24,12 & \multirow{2}{*}{30,87} \\
\hline \multirow[t]{3}{*}{ AML Sul } & 643106 & 9528,60 & 674,94 & 6,74 & \\
\hline & \multicolumn{5}{|c|}{ Área Líquida } \\
\hline & Habitantes & ha & Densidade Líquida $\left(\mathrm{hab} / \mathrm{km}^{2}\right)$ & Densidade Líquida (hab/ha) & Total AML \\
\hline AML Norte & 1874497 & 24110,44 & 7774,62 & 77,74 & \multirow{2}{*}{127,47} \\
\hline AML Sul & 643106 & 12934,20 & 4972,13 & 49,72 & \\
\hline
\end{tabular}


Da análise dos valores obtidos, e quando confrontados com as medidas de sustentabilidade apresentadas nas figuras 13 a 18, duas leituras podem ser feitas: uma mais directa, circunscrita aos quadros; e outra mais abrangente, com interpretação dos valores obtidos baseada no conhecimento empírico do território de estudo.

Uma área metropolitana de Lisboa desequilibrada no que respeita aos usos do solo, de acordo com as normas de classificação de (FABOS, 1985; cf. definição de área sustentável). Efectivamente, regista-se 69\% da área global da AML adstrita a usos de produção, e 50\% a usos de protecção, contra $19 \%$ classificados como áreas de impermeabilização. No que respeita ao cálculo das densidades, por forma a procurar georreferenciar e medir as áreas sujeitas a uma maior pressão horizontal, de acordo com o espectro de qualidade de vida de (SERRATOSA, 2000; cf. figura 3), concluiu-se que apenas as pessoas residentes no espaço determinado pela área líquida apresentam índices «razoáveis»(127hab./ha), enquanto os restantes denotam índices tidos como «bons» (<100hab./ha).

Quadro II - Valores das áreas e respectivas densidades populacionais, calculadas segundo a proposta da Carta de Ordenamento do PROTAML92.

Table II - Area dimensions and population densities, according to the Regional Plan for the Lisbon metropolitan area in 1992.

\begin{tabular}{|l|c|c|c|c|c|c|}
\cline { 2 - 7 } \multicolumn{1}{c|}{} & \multicolumn{6}{c|}{$\left(\mathbf{k m}^{\mathbf{2}}\right)$} \\
\cline { 2 - 8 } \multicolumn{1}{c|}{} & AML Norte & $\boldsymbol{\%}$ & AML Sul & $\mathbf{\%}$ & $\mathbf{A M L}$ & $\%$ \\
\hline Área de Produção & 762,8 & 66,5 & 999,3 & 76,0 & 1762,1 & 71,4 \\
\hline Área de Protecção & 321,5 & 28,0 & 374,6 & 28,0 & 696,1 & 28,2 \\
\hline Área Impermeabilizada & 399,7 & 34,8 & 312,4 & 24,0 & 712,1 & 28,9 \\
\hline Área Global (valor de referência) & $\mathbf{1 1 4 7}$ & $\mathbf{1 0 0}$ & $\mathbf{1 3 2 0}$ & $\mathbf{1 0 0}$ & $\mathbf{2 4 6 7}$ & $\mathbf{1 0 0}$ \\
\hline
\end{tabular}

\begin{tabular}{|c|c|c|c|c|c|}
\hline & \multicolumn{5}{|c|}{ Área Global } \\
\hline & Habitantes & ha & Densidade Global $\left(\mathrm{hab} / \mathrm{km}^{2}\right)$ & Densidade Global (hab/ha) & Total AML \\
\hline AML Norte & 1874497 & 114706,28 & 1634,17 & 16,34 & \multirow{2}{*}{21,21} \\
\hline \multirow[t]{3}{*}{ AML Sul } & 643106 & 132000,72 & 487,2 & 4,87 & \\
\hline & \multicolumn{5}{|c|}{ Área Bruta } \\
\hline & Habitantes & ha & Densidade Bruta $\left(\mathrm{hab} / \mathrm{km}^{2}\right)$ & Densidade Bruta (hab/ha) & Total AML \\
\hline AML Norte & 1874497 & 111521,84 & 1680,83 & 18,81 & \multirow{2}{*}{21,79} \\
\hline \multirow[t]{3}{*}{ AML Sul } & 643106 & 129055,60 & 498,32 & 4,98 & \\
\hline & \multicolumn{5}{|c|}{ Área Líquida } \\
\hline & Habitantes & ha & Densidade Líquida $\left(\mathrm{hab} / \mathrm{km}^{2}\right)$ & Densidade Líquida (hab/ha) & Total AML \\
\hline AML Norte & 1874497 & 39970,04 & 4689,76 & 46,90 & \multirow{2}{*}{67,48} \\
\hline AML Sul & 643106 & 31243,34 & 2058,37 & 20,58 & \\
\hline
\end{tabular}


Ainda numa leitura simples dos indicadores, revela-se um cenário consideravelmente «bom» ao nível metropolitano. No entanto, todos os que habitam, trabalham e vivem neste território sabem que existem problemas que estão camuflados ao abrigo de um ensaio científico experimental. Tal evidência obriga não só a confirmar os cálculos e a questionar o modelo, como também a considerar a hipótese de reafirmar o próprio modelo recorrendo a uma fonte oficial de dados sobre o uso do solo. O Quadro II mostra o resultado desta nova abordagem, que recorre à informação contida na carta de proposta de ordenamento incluída no relatório final do PROTAML de 1992.

Da leitura destes valores, enquanto indicadores de desenvolvimento sustentável do nosso modelo causal para a AML, sai reforçada a ideia, já constatada no exercício baseado no uso do solo de 1990, obtido por foto-interpretação, e exposto no Quadro 1: a AML denota «bons» índices de qualidade de vida (em consideração os valores das densidades populacionais pode verificar-se que todas as áreas se apresentam abaixo do índice 100), e um maior equilíbrio entre áreas de protecção e de impermeabilização ( $28 \%$ e $29 \%$, respectivamente), contra algum desfasamento face aos $71 \%$ de áreas de produção.

Não obstante este cenário, a realidade que se conhece é outra: diminuição dos espaços verdes e florestais; de consumo dos espaços agrícolas; de crescimento desgovernado das áreas urbanas; de densificação das áreas junto ao litoral; da subida das águas; do 'rasgar' do território por novas infra-estruturas rodoviárias em detrimento dos espaços públicos e de transportes alternativos; do desaparecimento das paisagens tradicionais, etc.

Nesta linha de raciocínio, a depuração e teste ao modelo tinham de ser feitas sobre uma sub-área da AML, cujos limites fossem recortados pelas áreas onde se verificasse a existência de maior densidade populacional ao nível da freguesia, segundo os dados estatísticos do Recenseamento Geral da População. Assim, recorreu-se à Base de Georreferenciação Estatística (BGRE91) e aos dados provisórios do Recenseamento Geral da População de 2001.

Seleccionou-se o conjunto de freguesias com mais de 1000 habitantes por $\mathrm{km}^{2}$ (fig. 10) e obteve-se, como resultado, um quantitativo de 133 freguesias num total de 216, o que equivale a $61 \%$ das freguesias da AML.

Pretende-se, com a criação desta área piloto da AML, confrontar a realidade teorizada e discutida por todos os membros da comunidade científica e política, e também pela população em geral, e a lógica da matemática e da estatística disponibilizada pelas TIG. Em resumo, procura testar-se a fidelidade do modelo. 


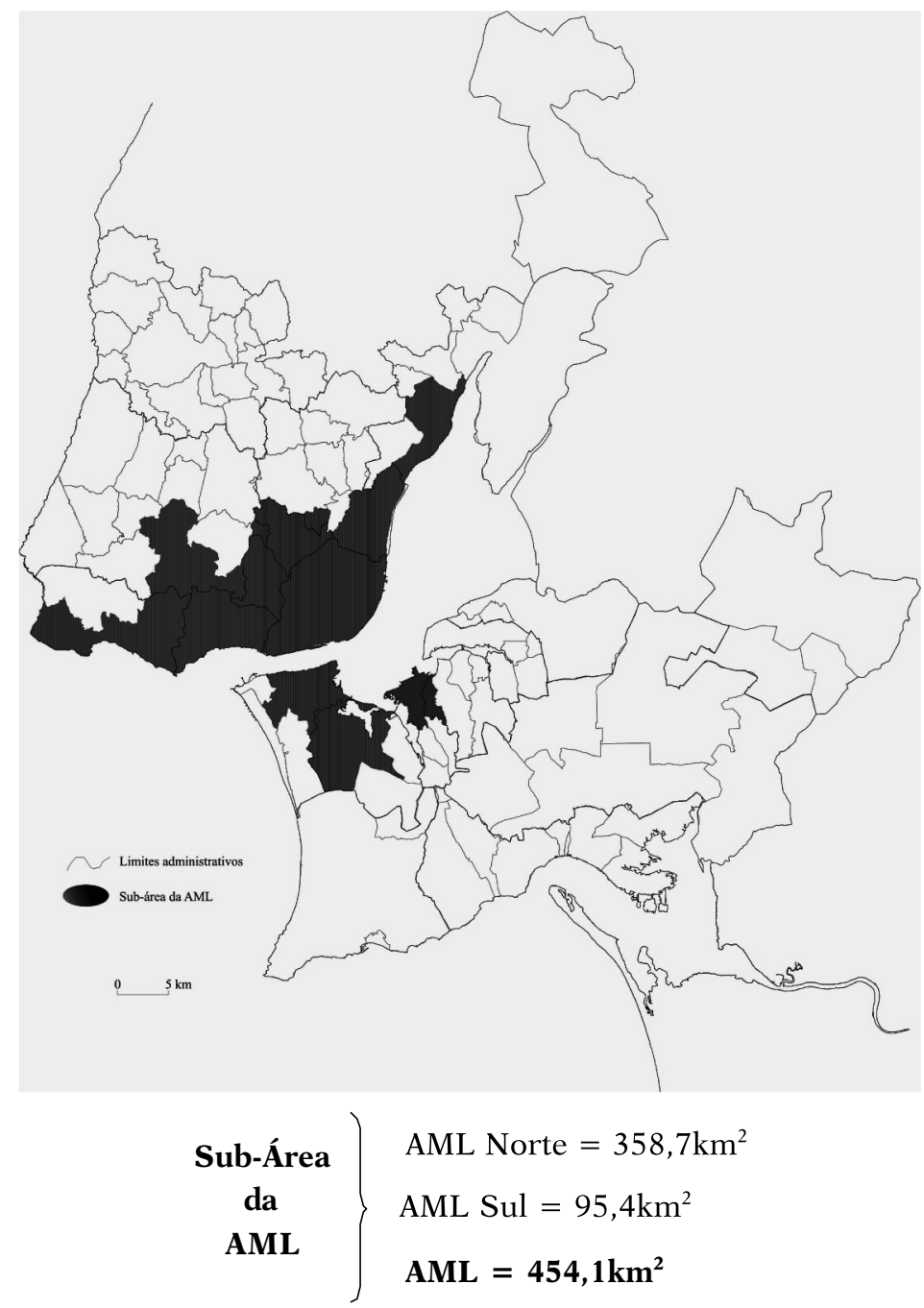

Fig. 10 - Sub-área da AML, correspondente às freguesias com densidade populacional superior a $1000 \mathrm{hab} . / \mathrm{km}^{2}$ (2001).

Fig. 10 -Lisbon's metropolitan sub-area, corresponding to the parishes with a population density grater than $1000 \mathrm{inb} . / \mathrm{km}^{2}$ (2001).

Da análise aos resultados decorridos da aplicação do modelo causal, para esta sub-área da AML, podem retirar-se várias ilações, nomeadamente no que respeita às margens Norte e Sul. No entanto, as primeiras avaliações devem restringir-se directamente aos indicadores de desenvolvimento sustentável apurados ao abrigo das teorias defendidas por FABOs e SERRATOSA e relativizadas aos 
valores obtidos, quer para o caso do CarTUS/AML de 1990, quer para o caso do uso do solo da proposta do PROTAML de 1992, para a totalidade do território da AML.

Nesta escala de valores e medidas de desenvolvimento sustentável, e numa primeira análise sobre os fenómenos georreferenciados para a sub-área da AML, tem-se a confirmação de um território desequilibrado (a teoria dos terços de FABOs não se verifica), conforme constatado para as outras situações. A novidade reside na dominância das áreas de impermeabilização relativamente às áreas de produção e de protecção. Efectivamente, enquanto nas outras duas situações dominavam as áreas de produção (Quadros I e II), para a sub-área da AML as áreas de impermeabilização ocupam por si só cerca de metade (49\%) do total do território; como aliás se pode confirmar pelo Quadro III.

Quadro III - Áreas, calculadas para a sub-área da AML, segundo o uso do solo de 1990.

Table III - Types of land use in the sub-areas of the Lisbon Metropolitan Area, 1990.

\begin{tabular}{|l|c|c|c|c|c|c|}
\cline { 2 - 8 } \multicolumn{1}{c|}{} & \multicolumn{6}{c|}{$\left(\mathbf{k m}^{2}\right)$} \\
\cline { 2 - 8 } \multicolumn{1}{c|}{} & AML Norte & $\%$ & AML Sul & $\%$ & AML & $\%$ \\
\hline Área de Produção & 97,4 & 35,2 & 34,0 & 46,0 & 131,4 & 27,0 \\
\hline Área de Protecção & 30,3 & 11,0 & 19,2 & 26,0 & 49,5 & 14,0 \\
\hline Área Impermeabilizada & 178,9 & 64,7 & 42,1 & 57,0 & 221 & 63,0 \\
\hline Área Global (valor de referência) & $\mathbf{2 7 6 , 5}$ & $\mathbf{1 0 0}$ & $\mathbf{7 4 , 3}$ & $\mathbf{1 0 0}$ & $\mathbf{3 5 0 , 8}$ & $\mathbf{1 0 0}$ \\
\hline
\end{tabular}

Numa segunda apreciação dos resultados apurados, mormente no que respeita aos indicadores de SERRATOSA, é de assinalar o significativo acréscimo das densidades populacionais para a sub-área da AML, facto que não é estranho dada a diminuição do espaço físico de concentração de população relativamente ao território a que foi aplicado o modelo, para as duas situações anteriores já descritas.

$\mathrm{Na}$ verdade, se nas outras situações os indicadores de densidade assinalavam bons e razoáveis níveis de qualidade de vida urbana (Quadros I e II), ao proceder-se à determinação da densidade desta nova área, para calibrar o modelo causal desenvolvido, verifica-se uma outra realidade: os indicadores revelam agora que a maioria da população urbana da AML está perto do limiar do valor que indicia «mau» nível de qualidade de vida, com particular destaque para a densidade líquida. Estes novos valores vêm comprovar a pertinência da aplicação do modelo causal à AML, assim como a adopção das escalas de medidas desenvolvidas e apresentadas por Fabos e SERRATOSA. 
Da análise e leitura conjunta destes indicadores (Quadro IV), para a sub-área da AML, fica o testemunho da realidade que se depreende e sente, sobretudo para aqueles que residem e/ou trabalham na área metropolitana de Lisboa.

Quadro IV - Valores das densidades populacionais, calculadas para a sub-área da AML, segundo o uso do solo de 1990.

Table IV - Land use area dimensions and population densities for the Lisbon metropolitan sub-area, 1990.

\begin{tabular}{|c|c|c|c|c|}
\hline & \multicolumn{4}{|c|}{ Área Global } \\
\hline & Habitantes & ha & Densidade Global (hab/ha) & Total AML \\
\hline AML Norte & 1874497 & 27651,90 & 67,80 & \multirow{2}{*}{154,30} \\
\hline \multirow[t]{3}{*}{ AML Sul } & 643106 & 7430,80 & 86,50 & \\
\hline & \multicolumn{4}{|c|}{ Área Bruta } \\
\hline & Habitantes & ha & Densidade Bruta (hab/ha) & Total AML \\
\hline AML Norte & 1874497 & 26650,70 & 70,30 & \multirow{2}{*}{157,40} \\
\hline \multirow[t]{3}{*}{ AML Sul } & 643106 & 7380,60 & 87,10 & \\
\hline & \multicolumn{4}{|c|}{ Área Líquida } \\
\hline & Habitantes & ha & Densidade Líquida (hab/ha) & Total AML \\
\hline AML Norte & 1874497 & 17891,00 & 104,80 & \multirow{2}{*}{257,30} \\
\hline AML Sul & 643106 & 4218,00 & 152,50 & \\
\hline
\end{tabular}

\section{Interface gráfico de utilização}

Numa tentativa de suscitar o interesse da comunidade para uma maior participação pública nos processos de tomadas de decisão, e assim procurar contribuir para o estreitamento do fosso entre comunidade científica e a sociedade em geral, criou-se aquilo que se designa por Interface Gráfico de Utilização.

A grande vantagem desta é o encapsulamento dos processos, ou seja, ao utilizador não é exigido um conhecimento sobre informática e programação, ou mesmo de operação do programa específico; basta-lhe saber qual o tema em questão e mediante as opções que o interface lhe disponibiliza (menus e botões), testar e verificar opiniões e resultados. Os processos desenrolam-se por ordem do utilizador, mas sem este os visualizar; apenas se lhe permite constar o resultado.

O interface gráfico de utilização 'encobre' os procedimentos atrás explanados, e desenvolvidos com recurso aos SIG, sintetizando-os mediante a visualização dos resultados indicadores do desenvolvimento sustentável para a AML. A este interface apelidou-se de medidor de impactes, e estrutura-se como se pode observar na figura 11. 


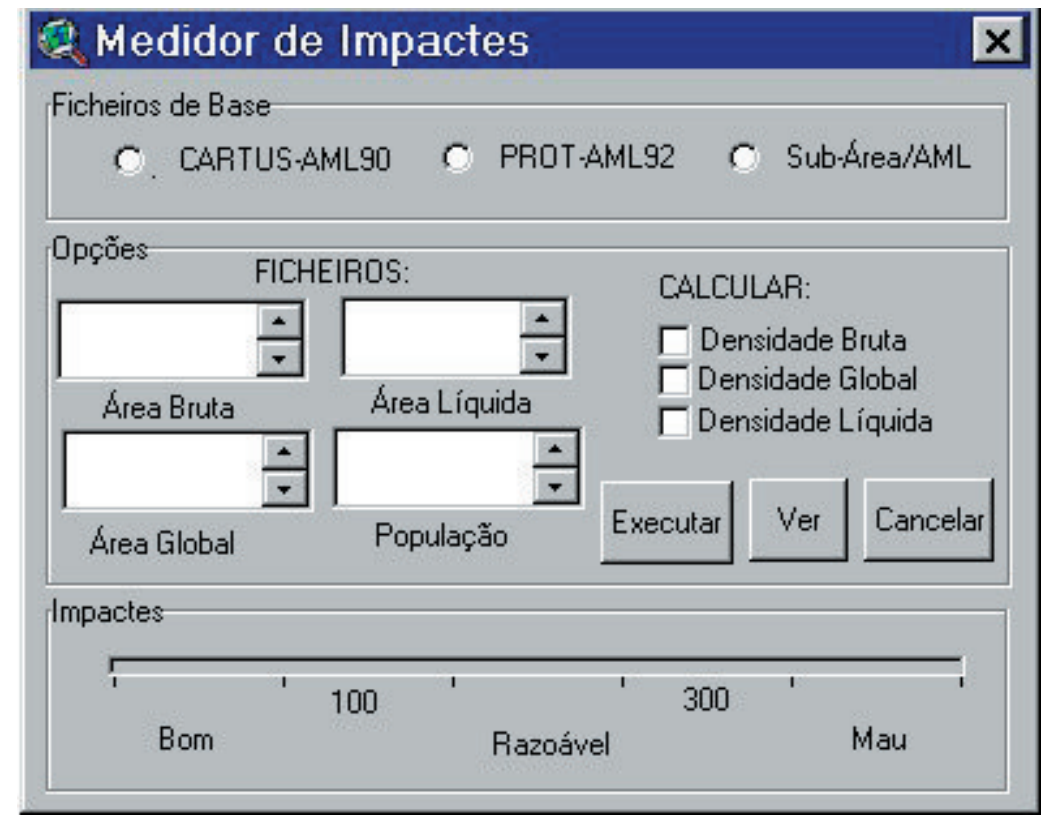

Fig. 11 - Interface gráfico do medidor de impactes.

Fig. 11 - Graphic user interface of the impact assessment software.

\section{CONCLUSÕES}

Tal como se verificou relativamente aos IDS (cf. I), também os modelos, por mais complexos que se apresentem ou estruturem, são sempre produto de uma generalização e simplificação do mundo conhecido. Todavia, é esta a forma que permite e facilita a lógica interna das construções teóricas e analíticas, viabilizando assim a monitorização dos sistemas, e o seu estudo evolutivo, ao mesmo tempo que contribui para uma ampla divulgação ao público, facilitando as tomadas de decisão, quer na acção-solução, quer na prevenção.

Assim, o modelo será sempre imperfeito, já que as considerações adoptadas têm associado um grau de artificialidade que mascara o comportamento real, inevitavelmente sujeito a variações.

Não obstante, o modelo causal apresentado vem demonstrar duas situações que se têm como verdadeiras:

- o exercício da modelação, suportado pelos avanços e aperfeiçoamentos das tecnologias e da técnica, constitui uma forma expedita e qualificada de estudo, análise e simplificação da realidade. Através dela é possível confrontar o histórico com o presente, assim como simular o futuro e avaliar tendências de uma forma causal, e em tempo útil. Um recurso 
indispensável ao conhecimento geográfico, e cada vez mais para as ciências, em geral.

- A AML, com base nos indicadores retirados da informação geográfica integrada e operada, acusa a juventude das políticas de ordenamento e planeamento do território ou a inexistência de uma figura de plano regional durante um período de 30 anos desde o Plano Director da Região de Lisboa, de 1964 (PDRL de 1964).

Efectivamente, a quantificação dos IDS a um território e a fenómenos geográficos concretos veio revelar aquilo que, teórica e empiricamente, já se sabia: a AML apresenta um desequilíbrio de usos/ocupação do solo. Assim definido o panorama e o contributo dos modelos, e deste modelo em particular, é pertinente colocar as questões: Então, qual a grande vantagem dos modelos? E para quê os SIG?

A grande vantagem das TIG em geral, e dos SIG em particular (produtos melhorados e sublimados da computação orientada para os fenómenos espaciais), reside na maior eficácia e gestão que se pode conferir à informação, assim como à optimização do conhecimento prévio para a produção de novos conhecimentos, sempre que a referenciação espacial é um requisito indispensável. Pode-se ainda dizer que a modelação geográfica veio permitir a exacta localização e identificação espacial dos problemas, e isolá-los ou enquadrá-los, simular possibilidades/hipóteses e apresentar soluções independentemente da escala. Por outro lado, permite uma abordagem dos IDS de forma quantificada, e de diagnósticos de erro calculado. Associada à computação, a modelação de sistemas complexos pode ser acessível ao público em geral.

A determinação da sub-área da AML (considerada no modelo causal) comprova na exacta medida estas afirmações, e os resultados daí retirados, devidamente identificados, constituem um alerta para baixos indicadores de qualidade de vida para o anel circunscrito à capital e radiado pelas principais infra-estruturas de transporte rodoviário e ferroviário, mais precisamente os eixos Lisboa-Vila Franca de Xira; Lisboa-Almada/Seixal/Moita; Lisboa-Sintra e Lisboa-Cascais.

Neste contexto, as TIG assumem-se como um meio incontornável para aplicar com eficácia as metodologias recomendadas pelas instituições e actores, assim como, e sobretudo, para a prática do ordenamento e planeamento do território. É do seu carácter operacional, e da sua faculdade de reunião de saberes, que se podem retirar dividendos positivos, no que concerne ao ordenamento no seu sentido mais lato, bem como ao exercício do poder (apoio à decisão) e participação da população, e à democratização da informação (disponibilização e livre acesso por todos, da informação concelhia). 


\section{BIBLIOGRAFIA}

Aronsson, T.; Johansson, P.; Lofgren, K., (2000) - Welfare, measurement, sustainability and green national accounting. A growth theoretical approach. Edward Elgar Publishing Limited.

Bossel, H. (1998) - Earth at a crossroads. Paths to a sustainable future. Cambridge University Press.

Ccrlvt, (2000) - Plano Regional de Ordenamento do Território da Área Metropolitana de Lisboa; Proposta. Comissão de Coordenação Regional de Lisboa e Vale do Tejo, Lisboa.

FABos, J. (1985) - Land-use planning. From global to local challenge. Chapman and Hall, New York and London.

Goodchild, M. (1992) - Geographical information science. International Journal of Geographical Information Systems 6(1): 31-45. [166].

Grueau, C. (1994) - Mise en ouvre d'un système d'information géographique pour la gestion municipale: Réalisation d'un prototype environnemental pour la ville de Lisbonne. Rapport de Stage. CNIG. Lisboa.

Longley, P.; Goodchild, M. et al. (2001) - Geographic information systems ad science. John Willey \& Sons., London.

Machado, J. R. (2000) - A emergência dos sistemas de informação geográfica na análise e organização do espaço. Dissertação de Doutoramento em Ciências do Ambiente na especialidade em Ordenamento e Território. FCG/UNL-FCT/MCT, Lisboa.

Martin, D. (1996) - Geographic information systems. Socioeconomic applications, 2. ${ }^{\mathrm{a}}$ ed. Routledge.

Matos, J. (2001) - Fundamentos de informação geográfica. Lidel, Lisboa.

Meadows, D. (1998) - Indicators and information systems for sustainable development. A report to the Balaton Group. Sustainability Institute.

Mopta (1995) - Guía para la elaboración de estudios del medio físico. Contenido y metodología. Secretaria de Estado de Medio Ambiente y Vivienda, Ministerio de Obras Publicas, Transportes y Medio Ambiente, Espanha.

Odum, E. (1971) - Fundamentos de Ecologia, 4. ${ }^{\text {a }}$ ed., FCG, Lisboa.

SARAIVA, G. (1999) - O rio como paisagem. Dissertação de Doutoramento em Arquitectura Paisagista. FCG/UNL-FCT/MCT, Lisboa.

Serratosa, A. (2000) - The city region in a world of globalisation: urban strategies for sustainable development. The strategic planning of Barcelona. Comunicação apresentada na INTA24 Annual Conference. Junho. Bergen.

Silva, E. A. (2002) - Beyond modelling in environmental and urban planning : planning support systems and the case study of Lisbon and Porto metropolitan areas, Portugal. Dissertação de Doutoramento submetida à Universidade de Massachusetts Amherst, em texto poli-copiado.

Telles, G. R. (2000) - PROTAML: um plano pouco ordenado. Revista Arquitectura e Vida; n. ${ }^{\circ} 1$. Lisboa: $38-43$.

Telles, G. R. (2000) - Por uma paisagem global. Revista Arquitectura e Vida; n. ${ }^{\circ}$ 3. Lisboa: 28-35.

W.C.E.D. (1987) - Our Common Future. World Commission on Environment and Development. Oxford University Press. 